\title{
The Search For Stellar Variability In M67: Ensemble Photometry with the Burrell Schmidt Telescope
}

\author{
Michael D. Joner, Eric G. Hintz, Thomas Stephens, Kenneth A. Nelson, \\ Helen Moore, and J. Ward Moody \\ Department of Physics and Astronomy, Brigham Young University, \\ Provo, Utah 84602, USA
}

\begin{abstract}
We have analyzed time-series data for 160 stars in the old open cluster M67. Approximately $400 \mathrm{CCD}$ frames from two nights on the Burrell Schmidt have been reduced differentially relative to an ensemble of seventeen stable stars. We present data on two new variables and evidence of four new suspected variable stars in M67.
\end{abstract}

\section{Introduction}

The old open cluster M67 has long been a favourite target for astronomers doing work with area detectors. Joner \& Taylor (1990) presented Cousins VRI photometry for nineteen standard stars in the M67 "dipper asterism". These stars are suitable for standardizing CCD images with an economy of effort. Other recent photometric results for the M67 cluster include Montgomery et al. (1993), as well as the works of Gilliland et al. (1991; hereafter G-91) and Gilliland \& Brown (1992). Montgomery et al. (1993) presented a deep $(V=20)$ survey of the central one-half degree of $\mathrm{M} 67$, which detailed fundamental cluster parameters. The work of Gilliland \& Brown (1992) and G-91 is especially relevant to the present investigation because, as part of a larger study investigating solar-like oscillations of M67 stars, it involved high-precision time-series photometry of M67. Due to the plate scale used by G-91, only a 6.6 arcmin field near the dipper asterism was observed. Furthermore, because G-91 were primarily interested in securing high-precision results on the faint stars contained in their sample, several of the bright giants and blue stragglers in the field were saturated.

We designed the present investigation to study the usefulness of a Schmidt telescope with a large format CCD in the discovery or observation of variable stars. Exposures were chosen so that the brightest cluster members would be well exposed on each frame without being saturated. In exchange for not saturating the brightest stars, we had to accept a limiting magnitude of about $\mathrm{V}=14.5$ before stellar variation became masked by observational error.

\section{Observations}

The data for this investigation were secured on two nights in February 1991 at Kitt Peak National Observatory. We utilized the Burrell Schmidt telescope equipped with the S2KA CCD. This combination yields a scale of $2.1 \mathrm{arcsec} / \mathrm{pixel}$. 
We thus found it possible to cover a 21 arcmin field by trimming the CCD frames to a 600 pixel square - this allowed us to use a portion of the S2KA that is free from column defects. Through the use of a subsection of the CCD, the readout overhead was cut to about 40 seconds. We used a standard Johnson V-filter for each frame and found that, in order to avoid saturating the brightest cluster members, the nominal exposure time was 25 seconds. We did increase exposures slightly for the higher airmass observations. Data were secured over periods of approximately six hours by following the cluster from about one hour east of the meridian to about five hours west of the meridian. An examination of the data reveals three or four breaks in the time-series each night; these were periods when Cousins $R$ and $I$ frames were secured as part of a future standardization project. The two nights used in this investigation were photometric and an examination of time-series extinction curves reveals no significant changes in sky conditions throughout the observation period, other than the expected decrease in intensity with increasing airmass.

\section{Data Analysis}

All frames were bias and overscan corrected using regular IRAF routines. We observed several twilight sky flats that were later processed and combined within IRAF and then applied to each of the cluster images. It is much easier to correct for flat-field variations when using only a subsection of the chip rather than the entire $2 \mathrm{~K}$ area. Because the scale of $2.1 \mathrm{arcsec} / \mathrm{pixel}$ and the excellent seeing conditions on the nights of the observations resulted in a very poorly sampled stellar point-spread function, we decided to do regular aperture photometry with the IRAF task APPHOT. After several tests, we selected an aperture of five pixels radius and a sky annulus three pixels wide. We also used the OFILTER option to enable a smooth sky fit. We found 140 stars brighter than fifteenth magnitude, which were well isolated from their nearest neighbour. By iterating through several comparison ensembles for the two nights' data, we were able to select seventeen stars that were very stable on both nights. These stars were then utilized as the standard comparison ensemble by differencing the instrumental magnitude for each program object with the ensemble mean for each frame. A plot of the error per observation against the standard V-magnitude for each star is displayed in Fig. 1. It should be noted that because we have several hundred frames for each object, the error of the mean is on the order of 0.003 magnitude for even the fifteenth-magnitude stars in our sample. For each star in our sample we plotted the differential ensemble magnitude versus heliocentric Julian date for each night of data.

We were able to "discover" variations with time for the well-known variable star AH Cancri, as well as the stars III-2 (found to be variable in G-91) and F 190 (confirmed variable in Gilliland \& Brown (1992)). F 190 appears to be a pulsating blue straggler with a fundamental period of approximately 89 minutes. Visual examination of light curve variations indicates the presence of additional pulsation modes. We also confirmed variability for the stars I-24 and F 131. I-24 is most likely a delta scuti variable with a period of approximately five hours. Further observations are needed in order to completely rule out the possibility that I-24 is an eclipsing binary. F 131 appears to be a low-amplitude pulsating 


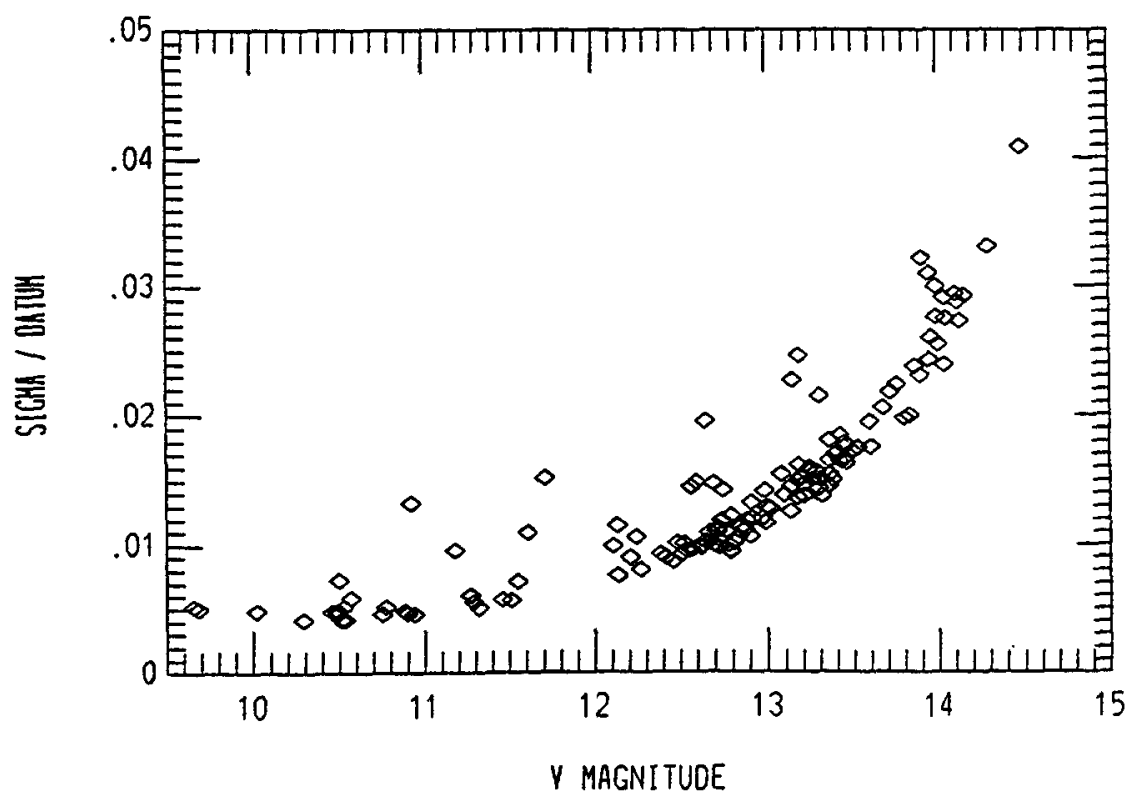

Figure 1. A plot of standard deviation per observation as a function of standard $\mathrm{V}$ magnitude for the stars studied in this investigation.
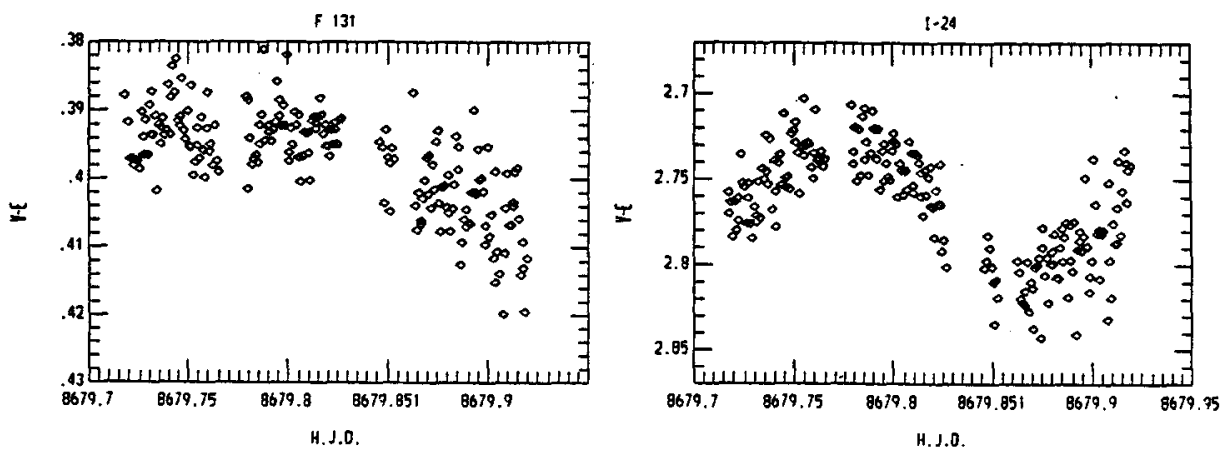

Figure 2. Displayed light variation for the newly discovered variable stars F 131 and I-24 in M67. 
blue straggler with an uncommonly long period (approximately 0.5 days). Data for the second night's observations of I-24 and F 131 are displayed in Fig. 2. We also suspect variations for four additional stars. However, further observations will be required to confirm the variations.

\section{Conclusions}

The Schmidt telescope has proven to be very successful in searching for and studying stellar variation over relatively large fields by utilizing differential ensemble reduction techniques. Two previously undiscovered variable stars have been found in the very well-observed old open cluster M67 using this technique.

We wish to thank the Department of Physics and Astronomy at Brigham Young University for the continued support that makes it possible for us to undertake these investigations. Thanks are also due to Lisa Joner for help during the preparation of this manuscript.

\section{References}

Gilliland R. L. \& Brown T. M., 1992, AJ, 103, 1945

Gilliland R. L., Brown T. M., Duncan D. R., Suntzeff N. B., Lockwood G. W., Thompson D. T., Schild R. E., Jeffrey W. A. \& Penprase B. E., 1991, AJ, 101, 541

Joner M. D. \& Taylor B. J., 1990, PASP, 102, 1004

Montgomery R. A., Marschall L. A. \& Janes K. A., 1993, AJ, 106, 181 